\title{
Trampas extracelulares de neutrófilos en sepsis
}

Neutrophil extracellular traps in sepsis

Armadilhas extracelulares de neutrófilos na sepse

Ansony Roger Godínez Vidal, ${ }^{*}$ Raúl Carrillo Esper, ${ }^{*}$ Ricardo Cabello Aguilera*

\section{RESUMEN}

Introducción: Los neutrófilos son uno de los componentes celulares más importantes de la inmunidad innata, debido a que estas células se reclutan rápidamente a los sitios de infección y pueden eliminar patógenos por múltiples métodos. En diferentes entidades, de entre las que se destaca la sepsis, los neutrófilos mejoran sus propiedades antimicrobianas mediante la liberación de trampas extracelulares de neutrófilos (TEN), constituidas por cromatina, histonas y proteínas granulares, proceso que es conocido por NETosis.

Objetivo: El objetivo de esta revisión es examinar los conceptos actuales, relacionados con los mecanismos subyacentes de la formación de TEN y su impacto en sepsis.

Conclusión: La desregulación de la NETosis causada por sepsis puede tener efectos deletéreos en sepsis, destacando inflamación, trombosis y disfunción multiorgánica.

Palabras clave: Neutrófilos, infección, cromatina, histonas, sepsis, inflamación.

\section{ABSTRACT}

Introduction: Neutrophils are one of the most important cellular components of innate immunity because these cells are rapidly recruited to sites of infection and can eliminate pathogens by multiple methods. In different entities from which sepsis disables neutrophils improve their antimicrobial properties by releasing neutrophils extracellular traps (NET), constituted by chromatin, histones and granular proteins, a process that is known by NETosis.

Objective: The objective of this paper is to review current concepts related to the underlying mechanisms of the formation of NETs, as well as the beneficial and harmful effects of them.

Conclusions: The dysregulation of NETosis caused by sepsis can have detrimental effects that cause inflammation, thrombosis and multi-organ failure. Keywords: Neutrophils, infection, chromatin, histones, sepsis, inflammation.

\section{RESUMO}

Introdução: Os neutrófilos são um dos componentes celulares mais importantes da imunidade inata, porque essas células se recrutam rapidamente para locais de infecção e podem matar patógenos por vários métodos. Em diferentes entidades das quais se destaca a sepse, os neutrófilos melhoram suas propriedades antimicrobianas liberando as armadilhas extracelulares de neutrófilos (del inglés: neutrophil extracelular traps, NET), compostas por cromatina, histonas e proteínas granulares, um processo conhecido como NETosis.

Objetivo: O objetivo desta revisão é revisar os conceitos atuais relacionados aos mecanismos subjacentes à formação das NET e seu impacto na sepse.

Conclusão: A desregulação da NETosis causada pela sepse pode ter efeitos deletérios na sepse, destacando inflamação, trombose e disfunção de múltiplos órgãos.

Palavras-chave: Neutrófilos, infecção, cromatina, histonas, sepse, inflamação.

\section{INTRODUCCIÓN}

Los neutrófilos son uno de los componentes celulares más importantes de la inmunidad innata, porque estas células se reclutan rápidamente a sitios de infección y

* HMG Hospital Coyoacán. Ciudad de México, México.

Abreviaturas: TEN $=$ Trampas extracelulares de neutrófilos. $\mathrm{MPO}=$ Mieloperoxidasa $P M A=12$-miristato de forbal-13. cfDNA = ADN libre de células. $E R O=$ Especies reactivas de oxígeno. LPS $=$ Lipopolisacáridos. $S$. aureus $=$ Staphylococcus aureus. $E$. coli $=$ Escherichia coli. DNasa $=$ Desoxirribonucleasa. CID = Coagulación intravascular diseminada.

Recepción: 24/04/2019. Aceptación: 17/08/2019.

www.medigraphic.com/medicinacritica pueden eliminar patógenos por diversos mecanismos. Los neutrófilos fagocitan los microorganismos en un proceso llamado fagocitosis. Luego, los microorganismos se encapsulan en fagosomas y son eliminados por nicotinamida adenina, mecanismos dependientes de dinucleótido fosfato oxidasa o por proteínas granulares antibacterianas como catepsinas, defensas, lactoferrina y lisozima (Figura 1). ${ }^{1}$

En el 2004, Brinkmann y colaboradores describieron un nuevo mecanismo antimicrobiano de neutrófilos, informando que la estimulación causa la extrusión de una red de fibras de cromatina recubiertas con proteínas antibacterianas como la elastasa, la catepsina G y la mieloperoxidasa (MPO). ${ }^{2}$ En ciertas condiciones, los neutrófilos mejoran sus propiedades antimicrobianas mediante la liberación de trampas extracelulares de neutrófilos (TEN), compuestas por cromatina extracelular decorada con histonas y numerosas proteínas granulares. Debido a que se consideró que la consecuencia final de este proceso es la muerte del neutrófilo, hacemos referencia a este mecanismo de muerte como «NETosis» (Figura 2). Sin embargo, el mecanismo detallado de la formación de TEN es aún desconocido. ${ }^{3}$ Muchos de estos componentes granulares, como la MPO, las defensas $\alpha$, la elastasa, la catepsina $G$ y la lactoferrina tienen actividades bactericidas capaces de eliminar microorganismos y/o sus factores de virulencia. La respuesta inflamatoria no controlada durante la sepsis es la causa subyacente propuesta de la formación excesiva de TEN. ${ }^{4,5}$

El aumento de la evidencia experimental y clínica indica que la formación excesiva de TEN durante la sepsis puede llevar al desarrollo de disfunción orgánica múltiple, lo que resalta el papel fisiopatológico de las TEN en la sepsis. ${ }^{6-9}$

El objetivo de este artículo es revisar los conceptos actuales relacionados con los mecanismos subyacentes de la formación de TEN y sus efectos en sepsis.

\section{Mecanismo de la NETosis}

Como células centinelas de inmunidad innata, los neutrófilos pueden responder a muchos patógenos o sus patrones moleculares están asociados mediante la liberación de TEN. El término «NETosis» comúnmente es usado para describir la secuencia de eventos celulares que condujeron a la liberación activa de TEN. 
Similar a otras formas de muerte celular, como la apoptosis o la muerte celular programada, una forma regulada de necrosis, la NETosis es un proceso altamente regulado. La desregulación de la NETosis, que se encuentra en muchos estados de enfermedad como la sepsis, puede provocar daños colaterales en el huésped. Sin embargo, los mecanismos celulares que promueven la liberación de las TEN siguen siendo poco conocidos. Brinkmann y su equipo, y Fuchs y otros documentaron por primera vez la NETosis in vitro en neutrófilos humanos al utilizar el potente activador de la proteína quinasa C, 12-miristato de forbal-13 (PMA). ${ }^{2}$

Tras la estimulación con PMA, los neutrófilos experimentan cambios morfológicos que incluyen la descondensación de la cromatina, la pérdida de la envoltura nuclear, la mezcla de contenidos nucleares y las proteínas granulares citoplasmáticas; también la pérdida de la integridad de la membrana y, en última instancia, la liberación de ADN libre de células (cfDNA). ${ }^{10}$

La muerte celular es inevitable en los neutrófilos sometidos a NETosis, ya que son incapaces de mantener un entorno intracelular constante sin una membrana celular intacta. Por esa razón, algunos investigadores describen este tipo de NETosis como «lítico» 0 «suicida». ${ }^{11}$

La generación de especies reactivas de oxígeno (ERO) por la NAPDH oxidasa es un proceso celular integral, pero no esencial en la NETosis. La NETosis suicida inducida por PMA y patógenos como Aspergillus fumigatus y Toxoplasma gondii son dependientes en la generación de ERO, que se produce corriente arriba de la proteína quinasa activada por mitógeno p38 y la fosforilación de la quinasa regulada por señal extracelular. ${ }^{12,13}$

Aún no está claro cómo la generación de ERO y sus efectos posteriores en última instancia conducen a la descondensación de la cromatina y la liberación de cfDNA. Papyannopoulos y colaboradores encontraron que la translocación de la elastasa de neutrófilos y los gránulos de MPO desde el citoplasma al núcleo es esencial para la descondensación de la cromatina durante la NETosis mediada por PMA. Este proceso parece ser independiente de las actividades enzimáticas de la elastasa de neutrófilos, pero el mecanismo molecular exacto responsable de esta translocación no está claro. ${ }^{14}$

Varios investigadores cuestionan la relevancia fisiológica de la NETosis mediada por PMA. ${ }^{15}$ En primer lugar, la NETosis inducida por PMA in vitro es un proceso que se desarrolla en horas, mientras que los neutrófilos in vivo fagocitan y se degranulan en cuestión de minutos después de encontrar microorganismos. En segundo lugar, algunos investigadores consideran que la NETosis inducida por PMA es «suicida», dado que los neutrófilos activados por PMA ya no pueden mantener la función celular normal después de la NETosis. Para comprender mejor la relevancia de NETosis in vivo, Yipp y su equipo visualizaron directamente los comportamientos de los neutrófilos dentro de las infecciones de la piel causadas por Staphylococcus aureus (S. aureus) en ratones y seres humanos mediante microscopia. Encontraron que los neutrófilos se someten a la descondensación de la cromatina y la liberación de ADN en cuestión de minutos después de encontrar $S$. aureus mientras mantienen una membrana celular intacta. Curiosamente, los neutrófilos que se encontraban en la red, ahora anucleares, continuaron realizando quimiotaxis y fagocitando bacterias cercanas. ${ }^{15}$ Este mecanismo único de NETosis, denominado NETosis «vital», se confirmó más tarde en los modelos sépticos murinos utilizando lipopolisacáridos (LPS) o Escherichia coli (E. coli). Además de mantener su capacidad funcional después de la NETosis vital, los neutrófilos deben liberar de alguna manera su ADN mientras preservan la integridad de la membrana celular. El laboratorio de Kubes respondió a esta pregunta demostrando que, en presencia de $S$. aureus, los neutrófilos humanos forman vesículas de la envoltura nuclear, que luego se fusionan con la membrana plasmática para liberar el ADN al espacio extracelular. ${ }^{16}$

\section{Beneficios de la NETosis}

Trampa microbiana y prevención de diseminación

En un modelo de ratón de fascitis necrosante, los ratones knockout para PAD4 con capacidad reducida para producir TEN fueron más susceptibles a la infección por Staphylococcus aureus, lo que sugiere que las TEN tienen un papel protector en la defensa contra los organismos invasores. ${ }^{17}$

Se ha demostrado que las TEN ejercen actividades antimicrobianas al atrapar físicamente o matar directamente los microorganismos. La evidencia más temprana de las propiedades antimicrobianas de las TEN se recopiló mediante microscopia electrónica de barrido de alta resolución; se incluyen los siguientes microorganismos: Shigella flexneri, S. aureus, Klebsiella pneumoniae, Candida albicans y Leishmania, los cuales se observaron físicamente unidos a los elementos estructurales de las TEN. La capacidad de las TEN para atrapar bacterias fue confirmada por Buchanan y otros, que utilizaron Streptococcus del grupo A que expresa desoxirribonucleasa (DNasa), en un modelo de ratón de fascitis necrosante. Se encontró que los ratones tratados con la cepa que expresa DNasa tenían lesiones cutáneas más importantes y diseminación bacteriana, lo que indica que las TEN no sólo mejoran la destrucción de bacterias sino que también atrapan a las bacterias para impedir su propagación. ${ }^{18}$ Esto fue confirmado por McDonald y colaboradores, quienes utilizaron $E$. coli marcada con fluorescencia para documentar la captura 


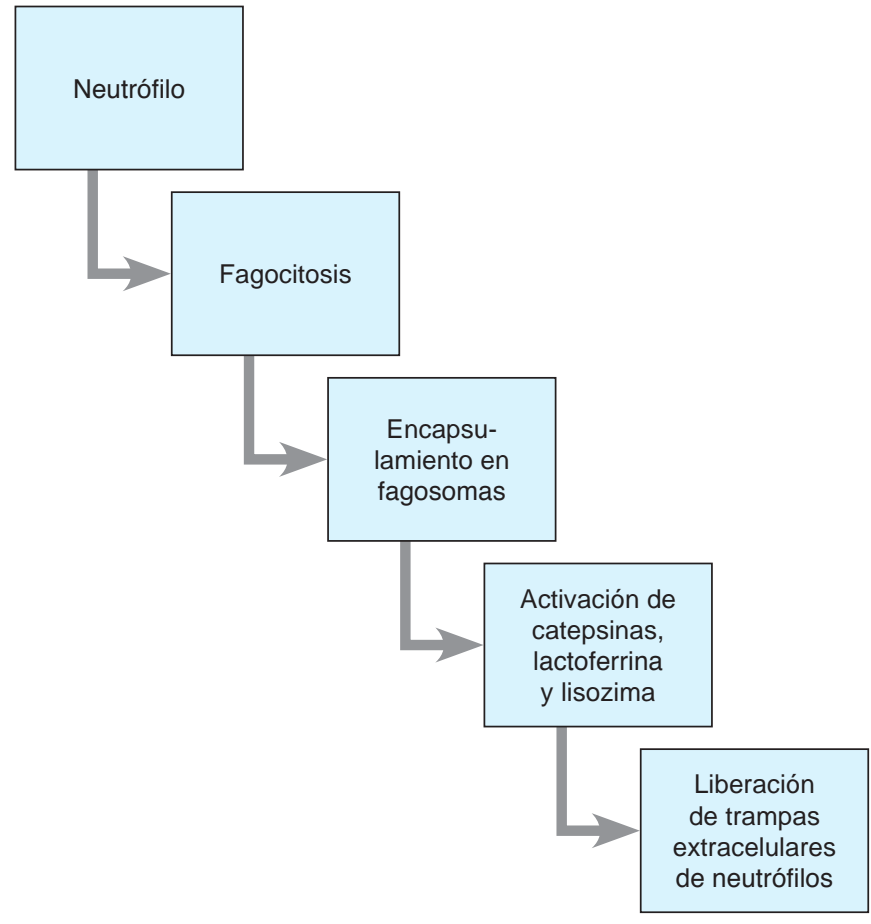

Figura 1: Fagocitosis. Los neutrófilos fagocitan los microorganismos en un proceso llamado fagocitosis. Luego, los microorganismos se encapsulan en fagosomas y son eliminados por nicotinamida adenina. Mecanismos dependientes de dinucleótido fosfato oxidasa o por proteínas granulares antibacterianas como catepsinas, defensas, lactoferrina y lisozima.

in vivo de bacterias dentro de los sinusoides del hígado tratados con LPS. ${ }^{19}$ El grupo también encontró que las TEN dentro de los sinusoides del hígado potencian la capacidad del hígado para atrapar bacterias una vez que las células de Kupffer se ven abrumadas por una bacteriemia extensa. Como se esperaba, los microorganismos que poseen la capacidad de descomponer rápidamente el ADN son más virulentos; por ejemplo, Streptococcus pneumoniae, una bacteria grampositiva que se encuentra comúnmente en el tracto respiratorio humano, puede expresar el factor de virulencia que degrada el ADN (endA), lo que permite que ocurra una bacteriemia y sepsis. ${ }^{20}$

\section{Actividad antimicrobiana directa}

En teoría, las TEN deben poseer propiedades antimicrobianas, ya que los componentes como las histonas, la catepsina G y la MPO ejercen actividades bactericidas. Sin embargo, la actividad antimicrobiana directa de las TEN in vivo es controvertida. Es probable que los datos publicados en conflicto reflejen los diferentes métodos utilizados para medir las propiedades antimicrobianas de las redes. ${ }^{21}$

La forma más directa de evaluar las propiedades microbicidas de las TEN in vitro es cultivar microbios y evaluar su viabilidad después de la incubación con neutrófilos formadores de TEN. Usando este método, los estudios encontraron que las TEN desmanteladas por digestión con ADNasa permitieron el crecimiento de $S$. aureus y Candida albicans atrapados. ${ }^{22}$ Una explicación para esto es que las proteasas granulares liberadas en el espacio extracelular son inactivadas rápidamente por el inhibidor de la proteinasa alfa-1. Las proteasas como elastasa de neutrófilos deben, por lo tanto, protegerse de los ácidos nucleicos para llevar a cabo sus actividades proteolíticas y microbicidas. Otro método utilizado es evaluar la viabilidad de los microbios en presencia de neutrófilos que no pueden producir TEN. Bianchi y su equipo mostraron que la falta de NETosis se asoció con el crecimiento de Aspergillus nidulans. ${ }^{23}$ Los componentes de las TEN también pueden minimizar la patogenicidad de los microbios al inactivar sus factores de virulencia. ${ }^{24}$

\section{TEN en sepsis}

Mientras que las TEN protegen al huésped al limitar el crecimiento y la diseminación microbiana, el exceso de NETosis durante la sepsis puede ser perjudicial para el huésped. Recientes descubrimientos en experimentos in vitro y modelos animales demostraron el papel crucial de las TEN en la patogénesis de la trombosis intravascular, la coagulación intravascular diseminada (CID) y la disfunción multiorgánica. ${ }^{25}$

\section{TEN $y$ trombosis en sepsis}

La inflamación sistémica y la liberación de citoquinas proinflamatorias durante la sepsis pueden provocar una activación anormal del sistema de coagulación. La trombosis excesiva se previene normalmente mediante la activación concurrente de las vías anticoagulantes que involucran al inhibidor de la vía del factor tisular, antitrombina, trombomodulina y proteína C. La activación continua de las vías de coagulación durante la sep-

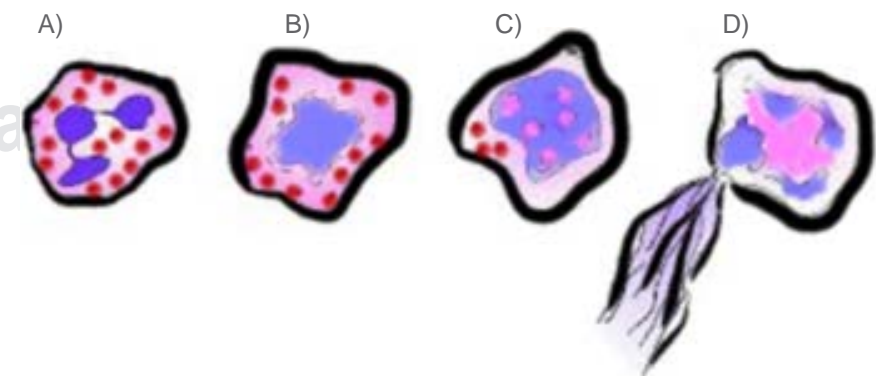

Figura 2: Proceso de la NETosis, en donde se puede observar: A) activación del polimorfonuclear, B) desarrollo del nucleosoma, C) nucleosoma, D) expulsión de material nuclear constituido por histonas y cromatina, lo que constituye la trampa extracelular de neutrófilos. 
sis desregula los sistemas anticoagulantes y provoca trombosis intravascular excesiva. En última instancia, el consumo excesivo de plaquetas y las proteínas de la coagulación dan como resultado una coagulopatía de consumo. La CID avanzada en pacientes sépticos generalmente se presenta como hemorragia o insuficiencia orgánica múltiple. Las TEN y sus componentes pueden exacerbar la CID al mejorar directamente la formación de coágulos ex vivo, la activación de plaquetas, así como la inhibición de las vías anticoagulantes. ${ }^{26,27}$

\section{CONCLUSIÓN}

La evidencia en la literatura indica que la NETosis es un mecanismo altamente conservado de inmunidad innata. La desregulación de la NETosis causada por la sepsis puede tener efectos perjudiciales que causan inflamación, trombosis y fallo multiorgánico. Los componentes de NETosis y TEN son objetivos terapéuticos potenciales para el tratamiento de la sepsis.

\section{BIBLIOGRAFÍA}

1. Kolaczkowska $E$, Kubes P. Neutrophil recruitment and function in health and inflammation. Nat Rev Immunol. 2013;13(3):159-175.

2. Brinkmann V, Reichard U, Goosmann C, Fauler B, Uhlemann Y, Weiss DS, et al. Neutrophil extracellular traps kill bacteria. Science. 2004;303(5663):1532-1535.

3. Brinkmann V, Zychlinsky A. Beneficial suicide: why neutrophils die to make NETs. Nat Rev Microbiol. 2007;5(8):577-582.

4. Keshari RS, Jyoti A, Dubey M, Kothari N, Kohli M, Bogra J, et al. Cytokines induced neutrophil extracellular traps formation: implication for the inflammatory disease condition. PLOS One. 2012;7(10):e48111.

5. Delgado-Rizo V, Martinez-Guzman MA, Iniguez-Gutierrez L, Garcia-Orozco A, Alvarado-Navarro A, Fafutis-Morris M. Neutrophil extracellular traps and its implications in inflammation: an overview. Front Immunol. 2017;8:81.

6. Dwivedi DJ, Toltl LJ, Swystun LL, Pogue J, Liaw KL, Weitz JI, et al. Prognostic utility and characterization of cell-free DNA in patients with severe sepsis. Crit Care. 2012;16(4):R151.

7. Kaplan MJ, Radic M. Neutrophil extracellular traps: doubleedged swords of innate immunity. J Immunol. J Immunol. 2012;189(6):2689-2695.

8. Mai SH, Khan M, Dwivedi DJ, Ross CA, Zhou J, Gould TJ, et al. Delayed but not early treatment with $\mathrm{DNase}$ reduces organ damage and improves outcome in a murine model of sepsis. Shock. 2015;44(2):166-172.

9. Czaikoski PG, Mota JM, Nascimento DC, Sonego F, Castanheira $\mathrm{FV}$, Melo $\mathrm{PH}$, et al. Neutrophil extracellular traps induce organ damage during experimental and clinical sepsis. PLoS One. 2016;11(2):e0148142.

10. Fuchs TA, Abed U, Goosmann C, Hurwitz R, Schulze I, Wahn V, et al. Novel cell death program leads to neutrophil extracellular traps. J Cell Biol. 2007;176(2):231-241.

11. Yipp BG, Kubes P. NETosis: how vital is it? Blood. 2013;122(16):2784-2794.

12. Itakura A, McCarty OJ. Pivotal role for the mTOR pathway in the formation of neutrophil extracellular traps via regulation of autophagy. A Am J Physiol Cell Physiol. 2013;305(3):C348-C354.

13. Keshari RS, Verma A, Barthwal MK, Dikshit M. Reactive oxygen species- induced activation of ERK and p38 MAPK mediates PMA-induced NETs release from human neutrophils. J Cell Biochem. 2013;114(3):532-540.
14. Papayannopoulos V, Metzler KD, Hakkim A, Zychlinsky A Neutrophil elastase and myeloperoxidase regulate the formation of neutrophil extracellular traps. J Cell Biol. 2010;191(3):677-691.

15. Yipp BG, Petri B, Salina D, Jenne CN, Scott BN, Zbytnuik LD, et al. Infection-induced NETosis is a dynamic process involving neutrophil multitasking in vivo. Nat Med. 2012;18(9):1386-1393.

16. Pilsczek FH, Salina D, Poon KK, Fahey C, Yipp BG, Sibley CD, et al. A novel mechanism of rapid nuclear neutrophil extracellular trap formation in response to Staphylococcus aureus. J Immunol. 2010;185(12):7413-7425.

17. Li P, Li M, Lindberg MR, Kennett MJ, Xiong N, Wang Y. PAD4 is essential for antibacterial innate immunity mediated by neutrophil extracellular traps. J Exp Med. 2010;207(9):1853-1862.

18. Buchanan JT, Simpson AJ, Aziz RK, Liu GY, Kristian SA, Kotb $M$, et al. DNase expression allows the pathogen group $A$ Streptococcus to escape killing in neutrophil extracellular traps. Curr Biol. 2006;16(4):396-400.

19. McDonald B, Urrutia R, Yipp BG, Jenne CN, Kubes P. Intravascular neutrophil extracellular traps capture bacteria from the bloodstream during sepsis. Cell Host Microbe. 2012;12(3):324-333.

20. Beiter K, Wartha F, Albiger B, Normark S, Zychlinsky A, Henriques-Normark B. An endonuclease allows Streptococcus pneumoniae to escape from neutrophil extracellular traps. Curr Biol. 2006;16(4):401-407.

21. Brinkmann V, Zychlinsky A. Neutrophil extracellular traps: is immunity the second function of chromatin? J Cell Biol. 2012;198:773-783.

22. Menegazzi R, Decleva E, Dri P. Killing by neutrophil extracellular traps: fact or folklore? Blood. 2012;119:1214-1216.

23. Bianchi M, Hakkim A, Brinkmann V, Siler U, Seger RA, Zychlinsky $A$, et al. Restoration of NET formation by gene therapy in CGD controls aspergillosis. Blood. 2009;114:2619-2622.

24. Weinrauch Y, Drujan D, Shapiro SD, Weiss J, Zychlinsky A. Neutrophil elastase targets virulence factors of enterobacteria. Nature. 2002;417:91-94.

25. Vincent JL, Moreno R, Takala J, Willatts S, De Mendonca A, Bruining $\mathrm{H}$, et al. The SOFA (sepsis-related organ failure assessment) score to describe organ dysfunction/failure. On behalf of the Working Group on Sepsis-Related Problems of the European Society of Intensive Care Medicine. Intensive Care Med. 1996;22:707-710.

26. Gould TJ, Vu TT, Swystun LL, Dwivedi DJ, Mai SH, Weitz JI, et al. Neutrophil extracellular traps promote thrombin generation through platelet-dependent and platelet-independent mechanisms. Arterioscler Thromb Vasc Biol. 2014;34:1977-1984.

27. Gould TJ, Vu TT, Stafford AR, Dwivedi DJ, Kim PY, FoxRobichaud AE, et al. Cell-free DNA modulates clot structure and impairs fibrinolysis in sepsis. Arterioscler Thromb Vasc Biol. 2015;35:2544-2553.

Protección de personas y animales. Los autores declaran que para esta investigación no se han realizado experimentos en seres humanos ni en animales.

Confidencialidad de los datos. Los autores declaran que han seguido los protocolos de su centro de trabajo sobre la publicación de datos de pacientes.

Derecho a la privacidad y consentimiento informado. Los autores declaran que en este artículo no aparecen datos de pacientes.

\section{Correspondencia:}

Ansony Roger Godínez Vidal

Calle Árbol del Fuego Núm. 80,

Col. El Rosario, 04380,

Alcaldía Coyoacán, Ciudad de México.

Tel: (55) 18908891

E-mail: ansony.rgv@gmail.com 